\title{
Type Synthesis and Analysis of Geared Linkages with Linear Actuation
}

\author{
E-C. LOVASZ*, K.-H. MODLER**, C. POP***, F. POP****, D. MĂRGINEANU*****, \\ I. MANIU******* \\ *Politehnica University of Timisoara, 1 Mihai Viteazu Blv., 300222 Timisoara, Romania, E-mail: erwin.lovasz@upt.ro \\ **Technischen Universität Dresden,01062 Dresden, Germany, E-mail: karl-heinz.modler@tu-dresden.de \\ ***Politehnica University of Timisoara, 1 Mihai Viteazu Blv., 300222 Timisoara, Romania, E-mail: cristian.pop@upt.ro \\ ****Politehnica University of Timisoara, 1 Mihai Viteazu Blv., 300222 Timisoara, Romania, E-mail: florina.pop@upt.ro \\ *****Politehnica University of Timisoara, 1 Mihai Viteazu Blv., 300222 Timisoara, Romania, E-mail: dan.margineanu@upt.ro \\ ******Politehnica University of Timisoara, 1 Mihai Viteazu Blv., 300222 Timisoara, Romania, E-mail: inocentiu.maniu@upt.ro \\ crossref http://dx.doi.org/10.5755/j01.mech.24.1.17534
}

\section{Introduction}

Linear actuators are frequently used in mechatronics devices for generating a translation input movement. The mechanisms using linear actuators should contain a minimum number of elements in order to obtain compact and market competitive products. This condition is satisfied by using a direct linear actuation, a slider-crank, an inverted slider crank, a double slider, but also in the case of geared five-bar linkages with linear actuation. The geared linkages with rotating input motion were studied in many researches, considering two structure types of geared linkages with serial and parallel connected gear train (s. Fig. 1).

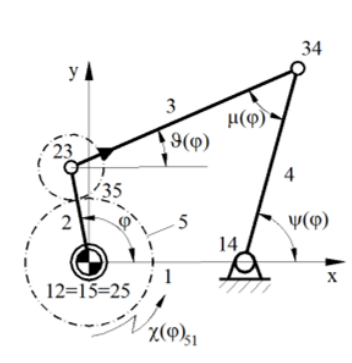

a

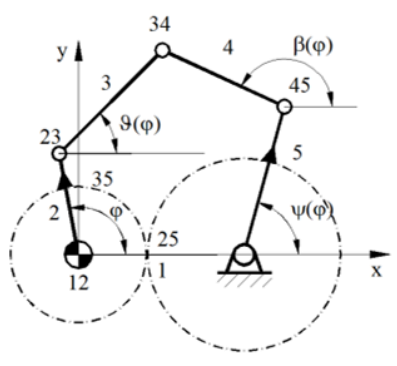

b
Fig. 1 Kinematic chains of the geared linkages with serial (a) and parallel (b) connected gear train

The geared linkages with serial connected gear train were studied beginning with Reuleaux [1] for some applications as sewing, stamping, steering and straight-line generators mechanisms. Freudenstein and Primerose studied in [2] the kinematical motion behaviour of the coupler point. Roth and Freudenstein [3] proposed a numerical synthesis method of the geared five-bar linkage for path generating tasks and Oleksa and Tesar [4] for the function generating tasks. The studies of this type of mechanisms were continued by Mundo et al. [5], [6] using non-circular gear train to connect the two links jointed in the frame and Parlaktas et. al. [7] developed a novel analysis method considering the expressions for the transmission angle.

The geared linkages with parallel connected gear train were studied by Neumann [8] as step mechanism with non-uniform continuous motion with high transmission ratio, with high swing angle [9] respectively with instantaneous dwell or pilgrim step [10], [11] and Hain [12]. Horani [13] and Rankers [14] developed analysis and synthe- sis methods for geared linkages with oscillating motion.

Only a few theoretical and experimental researches consider the geared five-bar linkages with parallel connected geared train and linear actuation. In [15] and [16] Lovasz et al presented a unitary developed analysis and synthesis methods for the geared linkages with linear actuation. Recently, Visa in [17] presented an approach for structural synthesis of planar geared linkages as multibody systems.

The aim of the paper is to perform an original type synthesis method and to show the relevant kinematic behaviour of the geared linkages with linear actuation and parallel connected gear train. As linear actuators, can be used pneumatic or hydraulic cylinders, linear electric motors, or screw type actuators.

\section{Type synthesis of the geared linkage with linear actuation and parallel connected chains}

The original proposed type synthesis method shows the systematic development of the possible kinematic chains and the mechanism structures of the geared linkages with linear actuation and parallel connected chains, based on the known structural relationships used in the mechanism science [15], [16].

The constrained motion condition can be expressed, according to Alt, through the equation:

$$
2 \cdot\left(e_{1}+e_{2} / 2\right)-3 \cdot n+3+M=0
$$

where: $e_{1}$ and $e_{2}$ represents the kinematic pairs number with $D o F=1$ respectively $D o F=2, n$ - elements number and $M-$ mechanism DoF.

In the considered case of geared linkages, the input conditions of the type synthesis for the kinematic chain considered at least the using of one kinematic pair with $D o F=2$ i.e. $e_{2}=1$ and the mechanism degree of freedom $D o F=1$. That means, taking into account the Eq. (1) yields the correlation between $n$ and $e_{1}$ and the condition to have integer solutions for them:

$$
n=\left(2 e_{1}+5\right) / 3, e_{1}=2+3 k, k(0,1, \ldots) .
$$

The first solution $k=0, e_{1}=2$ and $n=3$ shows the structure of a geared planetary mechanism, which is not convenient. The second solution for $k=1, e_{1}=5$ and $n=5$ lead to the geared linkages with minimal structure 
and parallel or serial connected chains. The required chains need to contain a prismatic kinematic pair, which should be the drive kinematic pair. The computation of the closed loops number for the planar kinematic chain follows with:

$$
N=\sum_{i=1}^{2} e_{i}-n+1
$$

that means for the geared linkages with linear actuation results $N=2$, where the computed $D o F$ for each loop is compulsory to be positive:

$$
M_{j}=3 \cdot(n+1)-2 \cdot e_{1}-e_{2}>0 .
$$

The numbers of the elements of different ranks satisfy the diophantine equations system:

$$
\left\{\begin{array}{l}
n=n_{2}+n_{3} \\
2 \cdot \sum_{i=1}^{2} e_{i}=2 \cdot n_{2}+3 \cdot n_{3}
\end{array},\right.
$$

where: $n_{2}, n_{3}$, represents the number of binary and ternary elements (higher ranks elements are not necessary). Taking into account the input conditions and the second solution of the Eq. (2) results as solution of the Eq. (5), the number of binary and ternary elements $n_{2}=3$ and $n_{3}=2$.

By a systematic development of all possible connections between the elements with kinematic pairs result a number of 11 possible kinematic chains. Computing the loops degree of freedom $M_{j}$, considering the compulsory condition (4) and adding the gear contact condition remain available only 6 kinematic chains. These kinematic chains are shown in Fig. 2.

In order to receive the useful geared linkages structures, it is necessary to apply the Reuleaux method for the 6 kinematic chains and the input criteria's:

- the drive should be the prismatic joint,

- the mechanism should not be consisting of serial connected chains,

- all the elements should lead to the motion transmission.

In Fig. 3 is shown an example for applying the Reuleaux method for the development of the geared linkage structure considering alternative one element as frame, one as drive and another as driven element.

From the resulted structures 4 structure of geared linkages mechanisms remain to be considered.

These will be classified and noted as:

- Planetary geared linkages, type Asi (Fig. 4),

- Cycloid geared linkages, type Zsi (Fig. 5).

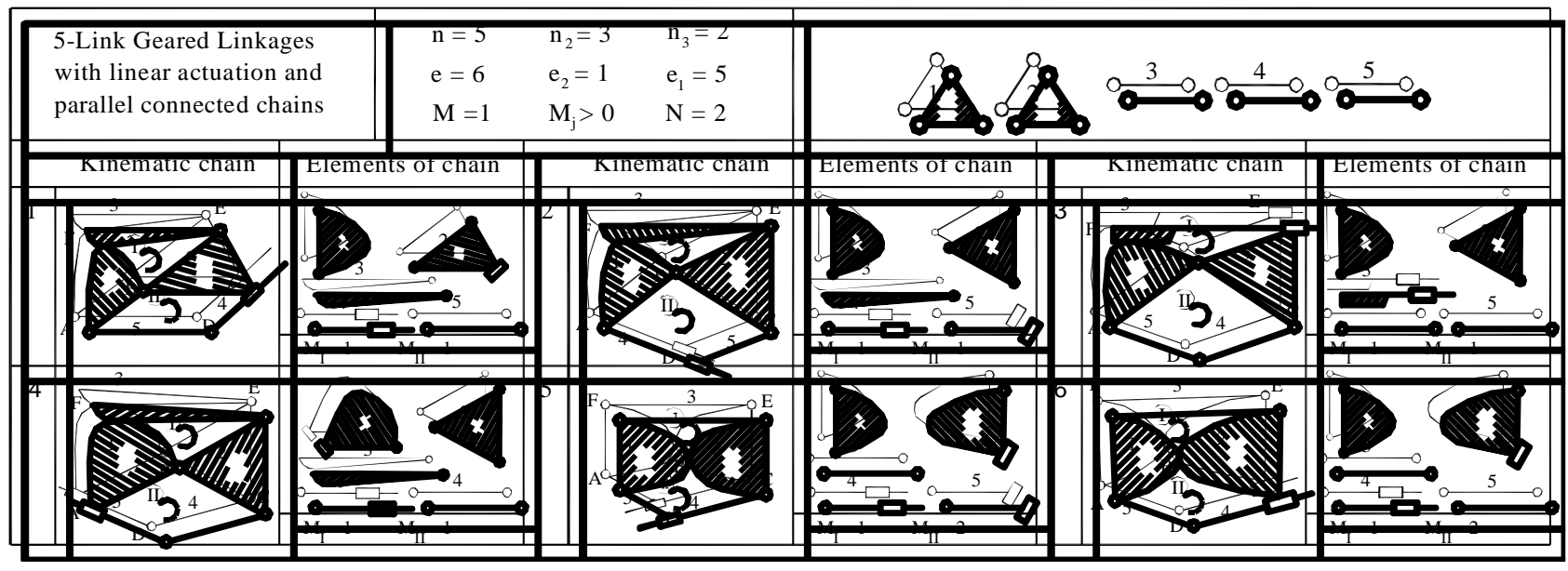

Fig. 2 Useful kinematic chains of the geared linkages with linear actuators

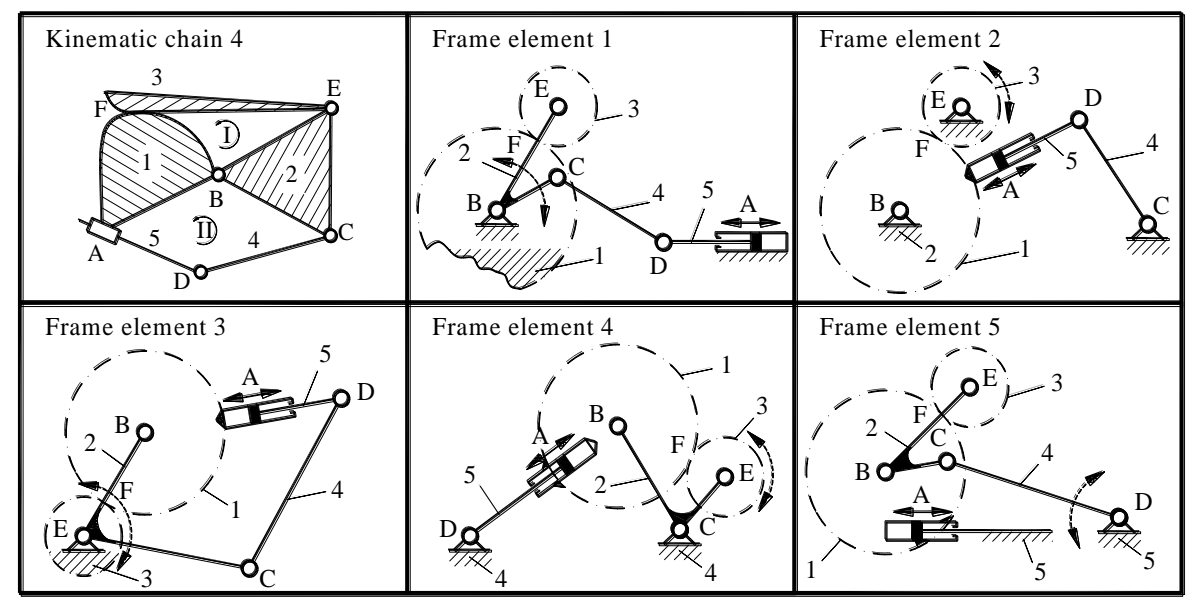

Fig. 3 Development by Reuleaux method of the geared linkages structures with linear actuators (case of chain 4) 


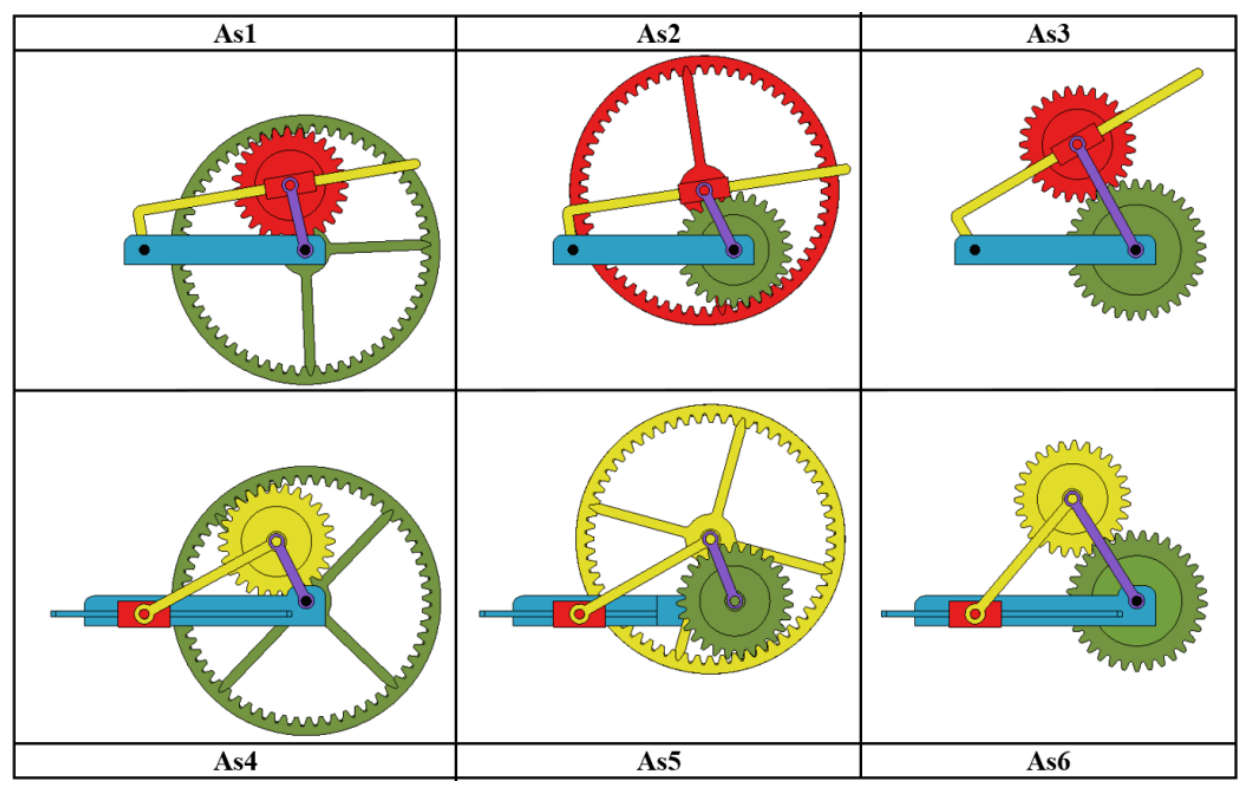

Fig. 4 Planetary geared linkages of type Asi

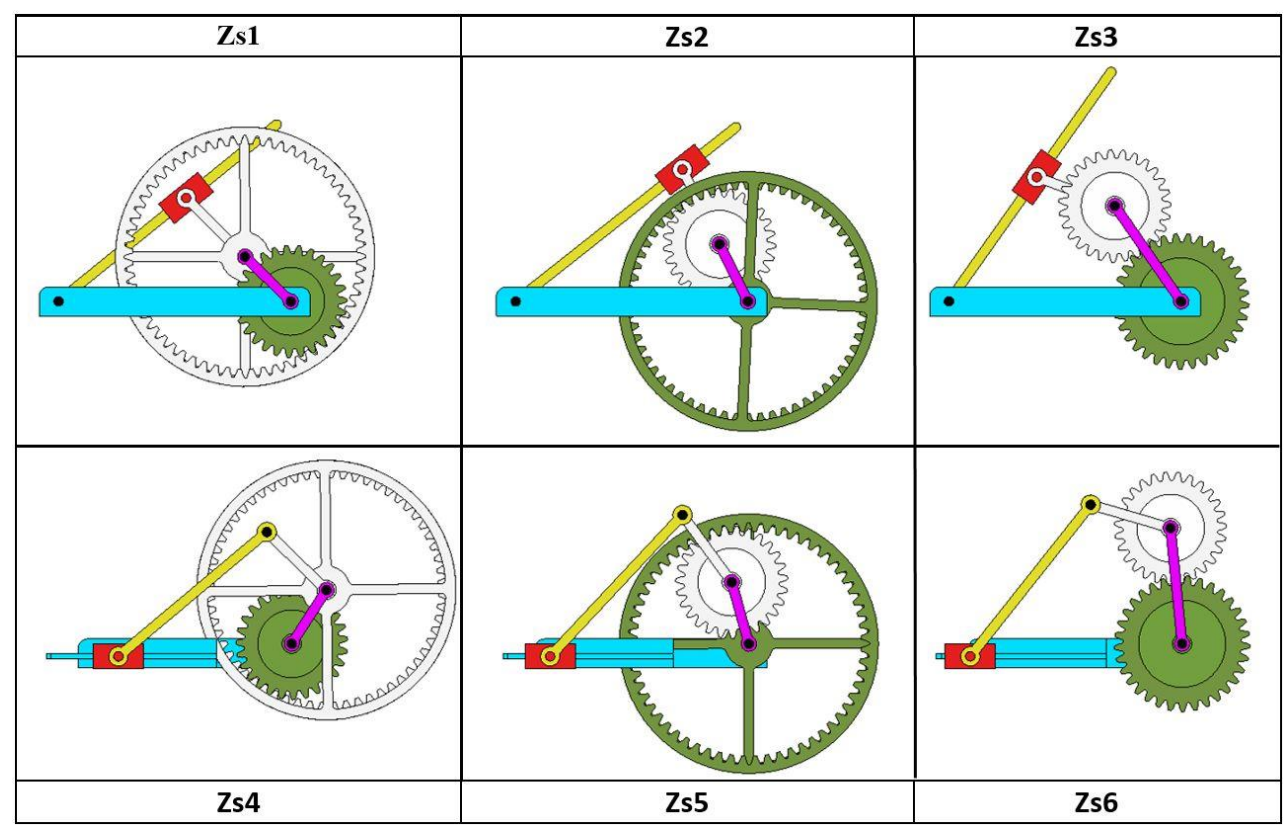

Fig. 5 Cycloid geared linkages of type Zsi

\section{Kinematic analysis of the geared linkages with linear} actuation and parallel connected chains

The kinematic analysis of planetary geared linkage with linear displacement actuator considers the mechanism consist of two base structures: a four-bar linkages and a planetary gear train (Figs. 5 and 6).

The transmission function zero order (TF-0 ord.) of the geared linkages follows in form [15]:

$$
\chi(s)=(1-\rho) \cdot \psi(s)+\rho \cdot \varphi(s),
$$

where: $\chi(s)$ is output angle or transmission function; $\rho= \pm r_{3} / r_{5}$ is gear ratio; $\varphi(s), \psi(s)$ is transmission functions of the base four-bar linkage.

The transmission functions $\varphi(s)$ and $\psi(s)$ of the four-bar linkages are to be determined by considering the closure loop vector equations (Table 1). The first order transmission function (TF-1 ord.) $\chi^{\prime}(s)$ and the second order transmission function (TF-2 ord.) $\chi^{\prime \prime}(s)$ can be computed in form:

$$
\left.\begin{array}{l}
\chi(s)=(1-\rho) \cdot \psi(s)+\rho \cdot \varphi(s) \\
\chi(s)=(1-\rho) \cdot \psi(s)+\rho \cdot \varphi^{\prime \prime}(s)
\end{array}\right\} .
$$

The angular velocity and acceleration result from the first and second derivative of the transmission function in respect with the time $t$ :

$$
\left.\begin{array}{l}
\dot{\chi}(t)=\chi(s) \cdot \dot{s}(t) \\
\ddot{\chi}(t)=\chi "(s) \cdot \dot{s}(t)+\chi(s) \cdot \ddot{s}(t)
\end{array}\right\},
$$

where: $\dot{s}=\dot{s}(t), \ddot{s}=\ddot{s}(t)$ are the input linear velocity and acceleration respectively, which are computed from dynamical analysis. 
Relationships for computing the transmission functions of the geared linkages [18]

\begin{tabular}{|c|c|}
\hline Geared linkages with inverted slider-crank as base structure & Geared linkages with slider-crank as basic structure \\
\hline 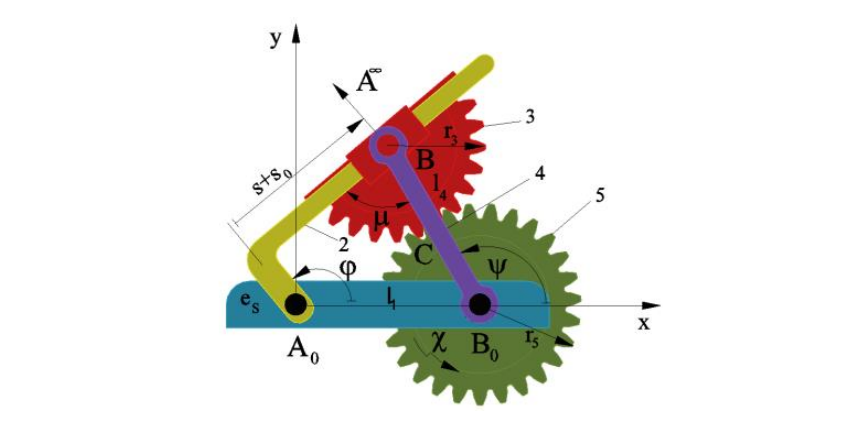 & stis \\
\hline$(e-i(s+s)) e=l+l e$ & $(e+i(s+s))+i l e=i l e$ \\
\hline \multicolumn{2}{|c|}{$\varphi(s)=2 \arctan ((B(s) \pm \sqrt{A(s)+B(s)-C(s)}) /(A(s)-C(s)))$} \\
\hline $\left.\begin{array}{l}\mathrm{A}(s)=2 l e, \quad B(s)=2 l(s+s) \\
C(s)=-l-e-(s+s)+l\end{array}\right\}$ & $\left.\begin{array}{l}A(s)=2 l(s+s), \quad B(s)=-2 l e \\
C(s)=e+(s+s)+l-l\end{array}\right\}$ \\
\hline$\psi(s)=\arccos \left(\left(e_{S}^{2}+\left(s_{0}+s\right)-l_{1}^{2}-l_{4}^{2}\right) / 2 l_{1} l_{4}\right)$ & $\psi(s)=\arcsin ((l \sin \varphi-e) / l)$ \\
\hline$\varphi(s)=\frac{l \sin \varphi(s)-(s+s)}{l(e \sin \varphi(s)-(s+s) \cos \varphi(s))}$ & $\varphi(s)=\frac{l \cos \varphi+(s+s)}{l((s+s) \sin \varphi+e \cos \varphi)}$ \\
\hline$\psi^{\prime}(s)=-\left(s_{0}+s\right) /\left(l_{1} l_{4} \sin \psi(s)\right)$ & $\psi(s)=-(l \cos \varphi \varphi) /(l \cos \psi)$ \\
\hline
\end{tabular}

\section{Kinematic behaviour of the geared linkages with linear actuation and inverted slider-crank as base structure}

In order to analyse the behaviour of the geared linkages with linear actuation and inverted slider-crank as base structure a numerical example with normalized values are used. The normalized geometrical parameters values range is given in the Table 2.

Table 2

Normalized geometric parameters

\begin{tabular}{|l|c|c|}
\hline Frame $\mathrm{A}_{0} \mathrm{~B}_{0}$ & $l_{1}$ & 1 \\
\hline Crank $\mathrm{B}_{0} \mathrm{~B}$ & $l_{4} / l_{1}$ & $0.5,0,2$ \\
\hline Eccentricity & $e_{S} / l_{1}$ & $-0.2,0,0.2$ \\
\hline Gear ratio & $\rho$ & $0.5,1,2$ \\
\hline
\end{tabular}

Fig. 6 shows the variation of transmission functions $\chi(s)$, considering the normalized crank values from Table 2 respectively the constant values for normalized eccentricity $e_{S} / l_{1}=0$ and the gear ratio $\rho=1$.

The transmission functions for the normalized crank values $l_{4} / l_{1} \leq 1$ shown for a large range an approximately linear variation and a very large oscillation angle. For the normalized crank values $l_{4} / l_{1}>1$ the first transmission function shows an approximately linear variation (Fig. 7).

The variation of the first transmission functions $\chi^{\prime}(s)$ shows a low influence for various normalized eccentricities $e_{S} / l_{1}$ given in Table 2 , constant normalized crank value $l_{4} / l_{1}=0.5$ and the gear ratio $\rho=1$ (Fig. 8).
The amplitude of the oscillation angle is also slightly influenced by changing the normalized eccentricity.

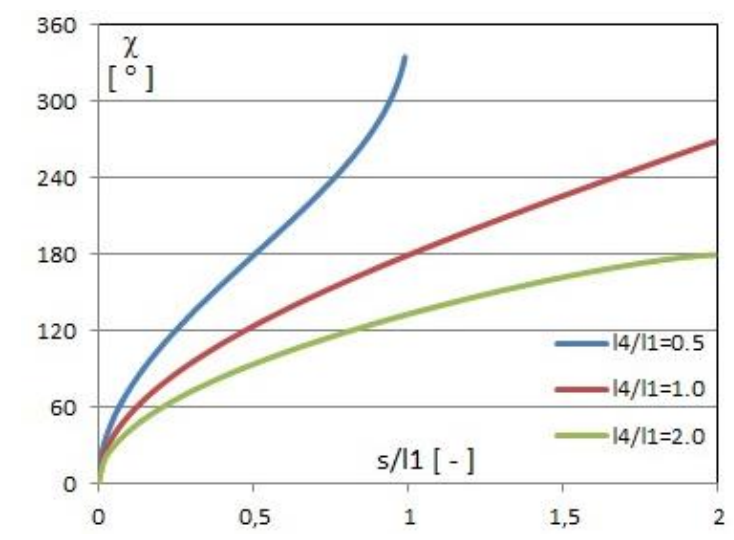

Fig. $6 \mathrm{TF}^{\prime} \mathrm{s}-0$ order $\chi(s)$ for various values of $l_{4} / l_{1}$

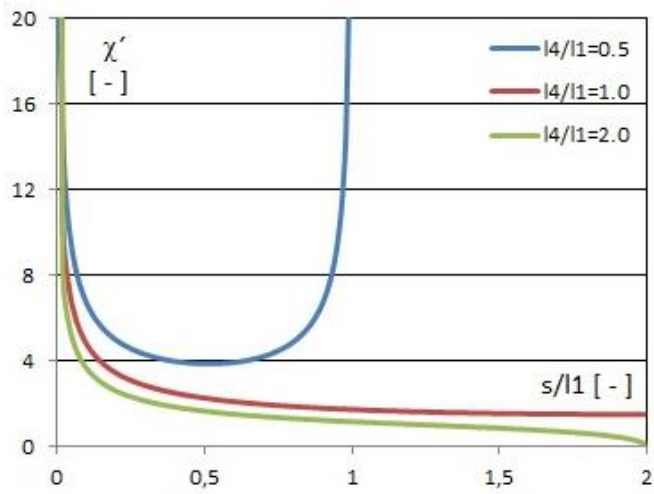

Fig. 7 TF's- 1 order $\chi^{\prime}(s)$ for various values of $l_{4} / l_{1}$ 
As expected, the variation of the transmission function $\chi(s)$ by increasing the gear ratio in the range given in Table 2 shows a very high oscillation angle of the output element (5) for the same normalized stroke of the actuator (Fig. 9).

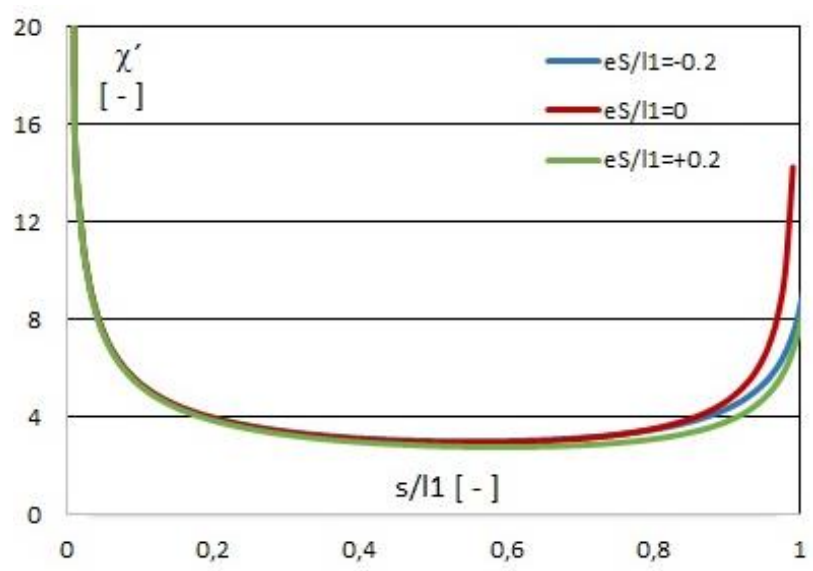

Fig. $8 \mathrm{TF}^{\prime} \mathrm{s}-1$ order $\chi^{\prime}(s)$ for various values of $e_{S} / l_{1}$

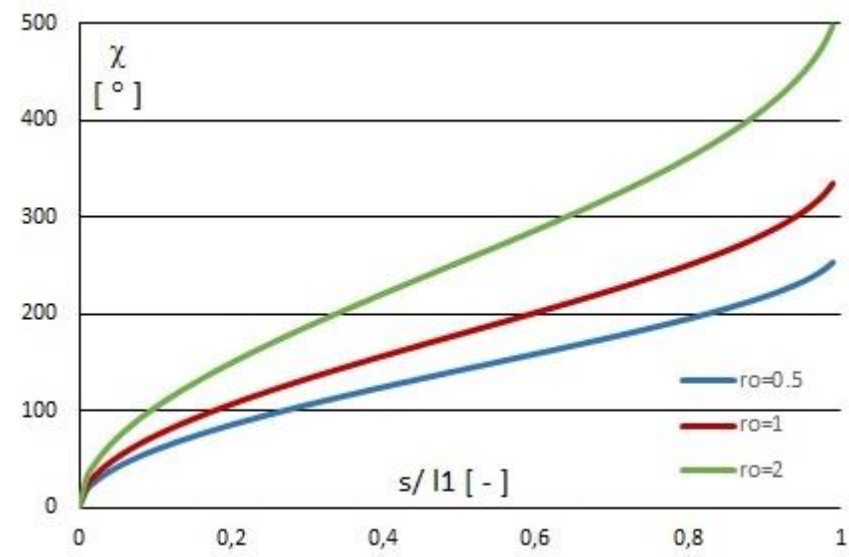

Fig. 9 TF's- 0 order $\chi(s)$ for various values of $\rho$

The variation of the first transmission functions increases the average values in approximately constant range by increasing the gear ratios. The computation of the first transmission functions $\chi^{\prime}(s)$, shown in Fig. 10, considers the constant normalized crank value $l_{4} / l_{1}=0.5$ and the normalized eccentricity value $e_{S} / l_{1}=0$.

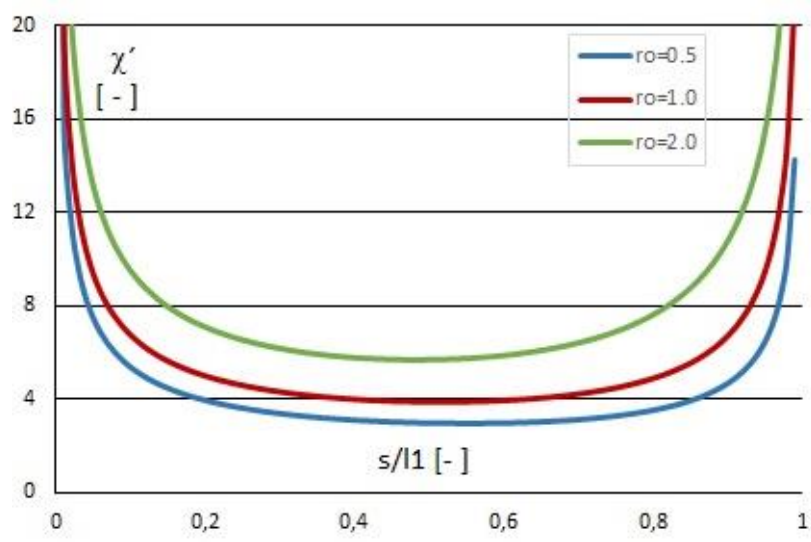

5. Kinematic behaviour of the geared linkages with linear actuation and slider-crank as base structure

The behaviour of the geared linkages with linear actuation and slider-crank as base structure is also exemplified by numerical examples with normalized values of the geometrical parameters given in the Table 3.

Fig. 11 shows the variation of the transmission functions $\chi(s)$ taking into account the normalized crank values from Table 3 respectively the constant values of normalized eccentricity $e_{S} / l_{3}=0$ and gear ratio $\rho=0.5$.

Table 3

Normalized geometric parameters

\begin{tabular}{|l|c|c|}
\hline Coupler $\mathrm{AB}$ & $l_{3}$ & 1 \\
\hline Crank $\mathrm{B}_{0} \mathrm{~B}$ & $l_{4} / l_{3}$ & $0.2,0.5,0.8$ \\
\hline Eccentricity & $e_{S} / l_{3}$ & $-0.2,0,0.2$ \\
\hline Gear ratio & $\rho$ & $0.5,1,2$ \\
\hline
\end{tabular}

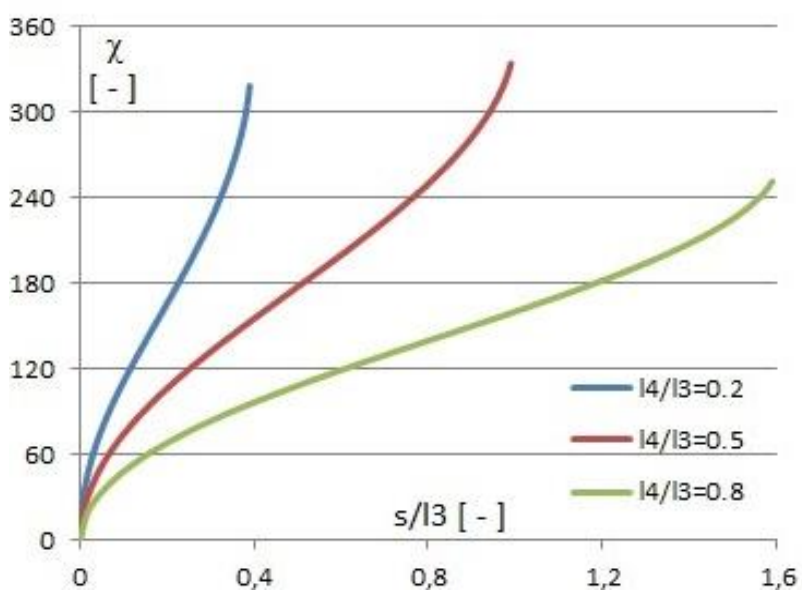

Fig. 11 TF's-0 order $\chi(s)$ for various values of $l_{4} / l_{3}$

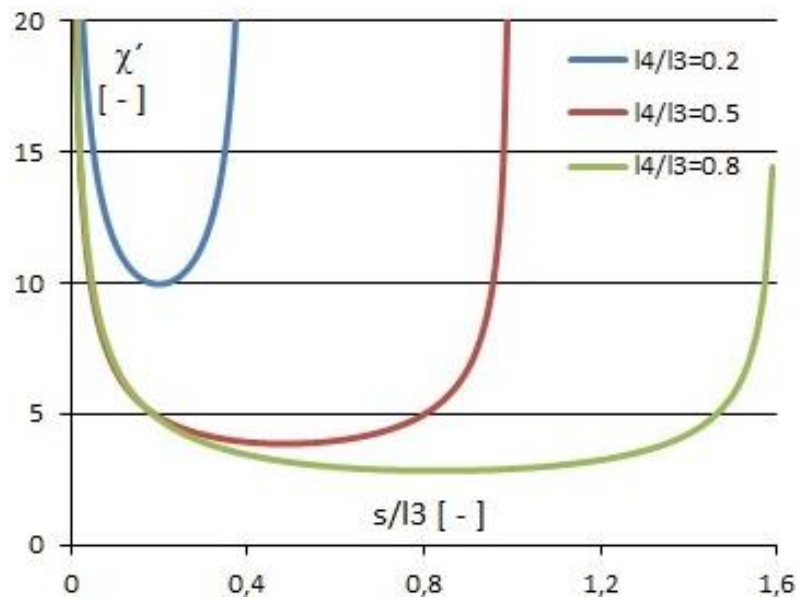

Fig.12 TF's-1 order $\chi^{\prime}(s)$ for various values of $l_{4} / l_{3}$

The transmission functions make sense only for the normalized crank values $l_{4} / l_{3} \leq 1$ and show also a large range with an approximately linear variation and a very large oscillation angle (Fig. 11 and Fig. 12).

Fig. $10 \mathrm{TF}^{\prime} \mathrm{s}-1$ order $\chi^{\prime}(s)$ for various values of $\rho$ 


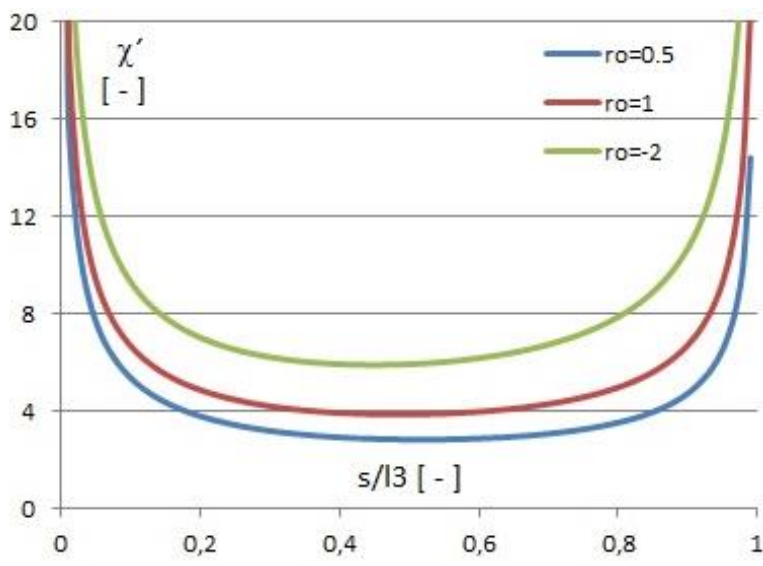

Fig.13 TF's-1 order $\chi^{\prime}(s)$ for various values of $\rho$

The variation of the first transmission functions $\chi^{\prime}(s)$ in between various normalized eccentricities $e_{S} / l_{3}$, given in Table 3 is similarly low influenced. Analogous, the variation of the transmission functions $\chi(s)$ and $\chi^{\prime}(s)$ by increasing the gear ratio in the range given in Table 3 shows a very high oscillation angle of the output element (5) and increased average values in approximately constant range.

\section{Conclusions}

The tape synthesis identifies 4 useful mechanism structures of the geared linkages with linear actuation and parallel connected chain and classifies them in two types: planetary geared linkages (Asi) and cycloid geared linkages (Zsi).

The kinematic analysis of the planetary geared linkages shows the capability to perform a very large oscillation angle with approximately constant ratio and proper transmission angle in defined motion range of the input element. Also it was shown the influence of the normalized geometrical parameters on the planetary geared linkage structures' behaviour.

Other expected advantages of this mechanism types consist in a very compact design and by using electric linear actuators with screw-nut for converting the rotational movement in translational movement the selflocking condition is fulfilled.

\section{References}

1. Reuleaux F. 1963. The kinematics of machinery. Dover, New York. 615 p.

2. Freudenstein F.; Primrose E.J.F. 1963. Geared five-bar motion. Trans. ASME, 85E, J. App. Mech. 30: 161-75. https://doi.org/10.1115/1.3636506.

3. Roth B.; Freudenstein F. 1963. Synthesis of pathgenerating mechanisms by numerical methods. Trans. ASME, J. Engng. Ind., 30: 298-305. https://doi.org/10.1115/1.3669870.

4. Oleksa S. A.; Tesar D. 1971. Multiply separated position design of the geared five-bar function generator. J. Eng. Ind 93(1), 74-84.: 298-305. http://dx.doi. org/10.1115/1.3427920.

5. Mundo D.; Gatti G. 2008. A graphical-analytical technique for the synthesis of noncircular gears in pathgenerating geared five-bar mechanisms, Trans. of the
CSME/de SCGM, 32 (3-4): 487-495.

6. Mundo D.; Gatti G.; Dooner D.B. 2009. Optimized five-bar linkages and non-circular gears for exact path generation, Mechanism and Machine Theory 44 (4): 751-760.

http://dx.doi.org/10.1016/j.mechmachtheory.2008.04.0 11.

7. Parlaktas V.; Soylemez E.; Tanik E. 2010. On the synthesis of a geared four-bar mechanism, Mechanism and Machine Theory 45(8): 1142-1152.

http://dx.doi.org/10.1016/j.mechmachtheory.2010.03.0 07.

8. Neumann R. 1977. Hochübersetzende Getriebe, Maschinenbautechnik, 26(7): 297-305.

9. Neumann R. 1985. Fünfgliedrige Räderkoppelgetriebe für große Schwingwinkel, Conference Dynamic und Getriebetechnik, TU Dresden.

10. Neumann R. 1986. Fünfgliedrige RäderkoppelSchrittgetriebe, Habilitation, TU Dresden.

11. Neumann R. 1987. Fünfgliedrigen RäderkoppelSchrittgetriebe, Aufbau, Synthese, Eigenschaften, Maschinenbautechnik, 36(10): 456-459.

12. Hain K. 1987. Zweiräder-Punktrasstgetriebe, Ind. Anz. 11: 23.

13. Horani M. 1977. Untersuchungen zur Analyse und Synthese zykloidengesteuerter Zweischläge, Dissertation, TU Dresden.

14. Rankers H. 1987. Rückkehrender Koppelrädermechanismus mit Doppelschleife, Konstruktion, 39(9): 335-357.

15. Lovasz E.-C.; Modler K.-H.; Hollman C. 2002. Auslegung der Räderkoppelgetriebe mit linearem Antrieb. 47. Internationales Kolloquium, TU Ilmenau, 23-26 September, (online).

16. Modler K.-H.; Hollmann C.; Lovasz E.-C.; Perju D. 2005. Geared Linkages with Linear Displacement Actuator Used as Function Generating Mechanisms, Proc. of the 11-th IFToMM World Congress, Tian Jin, 01.0405.04.2004, 1: 1254-1259.

17. Visa I.; Neagoe M.; Moldovan M.D. 2017. Structural synthesis of planar geared linkage mechanism as multibody systems, New Advances in Mechanisms, Mechanical Transmissions and Robotics, Mechanisms and Machine Science 46, Springer: 99-106. http://dx.doi.org/106.10.1007/978-3-319-45450-4_10.

\section{E-C. Lovasz, K.-H. Modler, C. Pop, F. Pop,} D. Mărgineanu, I. Maniu

\section{TYPE SYNTHESIS AND ANALYSIS OF THE GEARED LINKAGE WITH LINEAR ACTUATION}

S u m m a r y

The paper deals with the type synthesis of the geared linkages with linear actuation and parallel connected chain that identifies 4 useful mechanism structures of the geared linkages with linear actuation and classifies them in two types: planetary geared linkages (Asi) and cycloid geared linkages (Zsi).

For the planetary geared linkages (Asi) the mathematical models are developed and systematically presented. The obtained relationships are used for the kinematic 
analysis in order to investigate the behavior of planetary geared linkages. The kinematic analysis for various values of the normalized geometrical parameters shows their influence on the behaviour of these geared linkage structures. The analysis of the planetary geared linkages (Asi) pointed out their capability to perform a very large oscillation angle with approximately constant ratio and proper transmis- sion angle in defined motion range of the input element.

Keywords: geared linkage, linear actuation, type synthesis, kinematic analysis.

Received February 03, 2017

Accepted February 15, 2018 